\title{
On the Spectral Efficiency Maximization of Nonregenerative Cooperative MIMO Communication Systems
}

\author{
Fabien Héliot, Reza Hoshyar, and Rahim Tafazolli \\ Centre for Communication Systems Research, University of Surrey, \\ Guildford GU2 7HX, UK, Email: F.Heliot@Surrey.ac.uk
}

\begin{abstract}
Amplify-and-forward (AF) is one of the most common and simple approaches for transmitting information over a cooperative multi-input multi-output (MIMO) relay channel. It has recently been demonstrated that the spectral efficiency of AF scheme can be maximized by using the relay as a smart precoder. However, source node precoding has not been included in the overall maximization problem. In this paper, we propose a joint precoder design at the relay and source nodes for maximizing the cooperative mutual information (MI), i.e., the combination of direct and relay link MI, and thus, for further improving the spectral efficiency of AF scheme. In addition, we provide an algorithm for performing our joint precoding technique. Performance analysis indicates that our novel precoding algorithm outperforms other existing AF precoding methods in various link configurations.
\end{abstract}

\section{INTRODUCTION}

Cooperative communication is a well-documented research topic [1]-[6]. In a simple cooperation scenario, which is composed of a source node (SN), a single relay node (RN) and a destination node (DN), three main links are established, i.e., SN-DN, SN-RN and RN-DN links. The SN-DN link is refereed as the direct link and the combination of the $\mathrm{SN}$ RN and RN-DN links is known as the relay link. Several approaches have lately been followed to design cooperative communication systems and the most popular of them are decode and forward (DF) and amplify and forward (AF) [1][3] and [6]. DF is a regenerative approach where the source message is fully decoded and re-encoded at the RN and is then forwarded to the DN. On the contrary, AF is a nonregenerative approach where the $\mathrm{RN}$ simply amplifies the received signal from the $\mathrm{SN}$ and forwards it to the $\mathrm{DN}$.

In the traditional AF multi-input multi-output (MIMO) approach [6], the RN was first used as a simple equal gain (EG) amplifier. It has recently been demonstrated in [7] and [8] that AF scheme performance can be further enhanced by utilizing the $\mathrm{RN}$ as a smart precoder. In the case that both the channel state information (CSI) of the SN-RN and RN-DN links are available at the $\mathrm{RN}$, the power allocation techniques, which have been developed in [7] and [8], have shown to greatly enhance the spectral efficiency of AF by maximizing the relay link mutual information (MI) under a total power constraint. Later in [9], relay link MI performance has been further improved by performing a joint power allocation at the SN and RN when the direct link is weak. However, these techniques are not optimal in terms of cooperative MI, i.e., the combination of direct and relay link MI, since they do not take into account the CSI of the direct link. Here, we propose a joint power allocation algorithm for maximizing the cooperative MI of AF system by considering that full CSI (FCSI), i.e., the CSI of the three links, is available at the SN and RN nodes. Acquiring FCSI at the SN and RN nodes is hardly feasible in a real system, however, this assumption allows us to determine the maximum achievable cooperative MI performance of AF system for various link Signal-to-Noise Ratio (SNR) settings.

In this paper, we design a novel power allocation method for nonregenerative cooperative MIMO systems by relying on the system model that is introduced in Section II. In Section III, we provide an overview of the different types of power allocation methods that are based on MI maximization under a total power constraint. Then in Section IV, we explain how to jointly design the precoders at the $\mathrm{SN}$ and $\mathrm{RN}$ for maximizing the cooperative MI of AF when FCSI is available and provide an algorithm to do so. Simulation results in Section $\mathrm{V}$ indicate that our joint power allocation scheme outperforms the schemes of [7], [8] and [9] in various link SNR configurations. Finally, conclusions are drawn in Section VI.

\section{SySTEM MODEL}

We consider a system that is composed of three nodes, where a $\mathrm{SN}$, which is equipped with $n$ antennas, cooperate with a nonregenerative $\mathrm{RN}$, which is equipped with $q$ antennas, to transmit data to a $\mathrm{DN}$, which is equipped with $r$ antennas, as it is illustrated in Fig. 1.

For the simplicity of the introduction, we assume a half duplex relaying scenario with two phases of equal duration as in [7] and [8]. In the first phase, the SN broadcasts the signal $\mathbf{x}=\mathbf{R} \mathbf{s}$ to the $\mathrm{DN}$ and $\mathrm{RN}$; in the second phase, only the $\mathrm{RN}$ transmits to the DN. Note that $\mathbf{R} \in \mathbb{C}^{n \times n}$ is the $\mathrm{SN}$ precoding matrix and $\mathrm{E}\left\{\mathbf{s s}^{\dagger}\right\}=\mathbf{I}_{n}$, where $\mathbf{I}_{n}$ is a $n \times n$ identity matrix and $\mathrm{E}\{$.$\} stands for the expectation.$ The signal $\mathbf{x}$ is received by the DN as $\mathbf{y}_{0}=\mathbf{H}_{0} \mathbf{x}+\mathbf{n}_{0}$ and by the $\mathrm{RN}$ as $\mathbf{y}_{1}=\mathbf{H}_{1} \mathbf{x}+\mathbf{n}_{1}$ at the end of the first phase, where $\mathbf{H}_{0} \in \mathbb{C}^{r \times n}$ and $\mathbf{H}_{1} \in \mathbb{C}^{q \times n}$ characterize the MIMO channels of the SN-DN and SN-RN links, respectively. During the second phase, the signal $\mathbf{y}_{1}$ is amplified by using the precoding matrix $\mathbf{G} \in \mathbb{C}^{q \times q}$ at the $\mathrm{RN}$, is then transmitted 


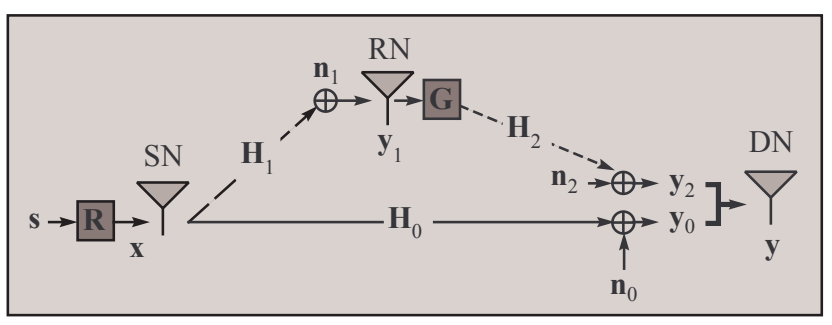

Fig. 1. Nonregenerative cooperative MIMO communication system model.

towards the DN and is received as $\mathbf{y}_{2}=\mathbf{H}_{2} \mathbf{G} \mathbf{y}_{1}+\mathbf{n}_{2}$ by the DN, where $\mathbf{H}_{2} \in \mathbb{C}^{r \times q}$ characterizes the MIMO channel of the RN-DN link. In addition, each of the channel matrices $\mathbf{H}_{0}, \mathbf{H}_{1}$ and $\mathbf{H}_{2}$ is a random matrix having independent and identically distributed (i.i.d.) complex Gaussian entries with zero-mean and unit variance. Furthermore, $\mathbf{n}_{0} \in \mathbb{C}^{r \times 1}$, $\mathbf{n}_{1} \in \mathbb{C}^{q \times 1}$ and $\mathbf{n}_{2} \in \mathbb{C}^{r \times 1}$ are vectors of independent zeromean complex Gaussian noise entries with a variance of $\sigma_{0}^{2}$, $\sigma_{1}^{2}$ and $\sigma_{2}^{2}$, respectively. The system model of the cooperative MIMO communication system that is depicted in Fig. 1 can be summarized as

$$
\mathbf{y}=\left[\begin{array}{l}
\mathbf{y}_{0} \\
\mathbf{y}_{2}
\end{array}\right]=\left[\begin{array}{c}
\mathbf{H}_{0} \\
\mathbf{H}_{2} \mathbf{G H}_{1}
\end{array}\right] \mathbf{x}+\left[\begin{array}{ccc}
\mathbf{I}_{r} & \mathbf{0} & \mathbf{0} \\
\mathbf{0} & \mathbf{H}_{2} \mathbf{G} & \mathbf{I}_{r}
\end{array}\right]\left[\begin{array}{l}
\mathbf{n}_{0} \\
\mathbf{n}_{1} \\
\mathbf{n}_{2}
\end{array}\right] .
$$

The cooperative MI that is shared between the transmit signal $\mathbf{s}$ and the receive signal $\mathbf{y}$ is accordingly expressed as [10]

$$
I(\mathbf{y} ; \mathbf{s})=\frac{1}{2} \log _{2}\left|\mathbf{I}_{2 r}+\mathbf{H R R}^{\dagger} \mathbf{H}^{\dagger} \mathbf{R}_{\mathbf{n}}^{-1}\right|=\frac{1}{2} \log _{2}\left|\begin{array}{cc}
\mathbf{A} & \mathbf{D} \\
\mathbf{C} & \mathbf{B}
\end{array}\right|,
$$

where the factor $1 / 2$ accounts for the two-phase transmission, $(.)^{\dagger}$ denotes the conjugate transpose operator, $\mathbf{H} \in \mathbb{C}^{2 r \times n}$ characterizes the cooperative MIMO channel and $\mathbf{R}_{\mathbf{n}} \in$ $\mathbb{C}^{2 r \times 2 r}$ is the aggregate noise covariance matrix. Moreover, the matrices $\mathbf{A}, \mathbf{B}, \mathbf{C}$ and $\mathbf{D}$ are given by

$$
\begin{aligned}
& \mathbf{A}=\mathbf{I}_{r}+\mathbf{H}_{0} \mathbf{R} \mathbf{R}^{\dagger} \mathbf{H}_{0}^{\dagger} \mathbf{R}_{\mathbf{n}_{0}}^{-1} \\
& \mathbf{B}=\mathbf{I}_{r}+\mathbf{H}_{2} \mathbf{G}\left(\mathbf{R}_{\mathbf{y}_{1}}-\mathbf{R}_{\mathbf{n}_{1}}\right) \mathbf{G}^{\dagger} \mathbf{H}_{2}^{\dagger} \mathbf{R}_{\mathbf{n}_{2}}^{-1}, \\
& \mathbf{C}=\mathbf{H}_{2} \mathbf{G} \mathbf{H}_{1} \mathbf{R} \mathbf{R}^{\dagger} \mathbf{H}_{0}^{\dagger} \mathbf{R}_{\mathbf{n}_{0}}^{-1} \\
& \mathbf{D}=\mathbf{H}_{0} \mathbf{R} \mathbf{R}^{\dagger} \mathbf{H}_{1}^{\dagger} \mathbf{G}^{\dagger} \mathbf{H}_{2}^{\dagger} \mathbf{R}_{\mathbf{n}_{2}}^{-1}
\end{aligned}
$$

where $\mathbf{R}_{\mathbf{n}_{0}}=\sigma_{0}^{2} \mathbf{I}_{r}, \mathbf{R}_{\mathbf{n}_{2}}=\sigma_{2}^{2} \mathbf{I}_{r}+\mathbf{H}_{2} \mathbf{G R}_{\mathbf{n}_{1}} \mathbf{G}^{\dagger} \mathbf{H}_{2}^{\dagger}, \mathbf{R}_{\mathbf{n}_{1}}=$ $\sigma_{1}^{2} \mathbf{I}_{q}$ and $\mathbf{R}_{\mathbf{y}_{1}}=\mathrm{E}\left\{\mathbf{y}_{1} \mathbf{y}_{1}^{\dagger}\right\}=\mathbf{R}_{\mathbf{n}_{1}}+\mathbf{H}_{1} \mathbf{R} \mathbf{R}^{\dagger} \mathbf{H}_{1}^{\dagger}$ is the relay received signal covariance matrix. The direct and relay link MI, i.e., $I\left(\mathbf{y}_{0} ; \mathbf{s}\right)$ and $I\left(\mathbf{y}_{2} ; \mathbf{s}\right)$, can also be computed by employing (2) for $\mathbf{H}=\mathbf{H}_{0}, \mathbf{R}_{\mathbf{n}}=\mathbf{R}_{\mathbf{n}_{0}}$ and $\mathbf{H}=\mathbf{H}_{2} \mathbf{G H}_{1}$, $\mathbf{R}_{\mathbf{n}}=\mathbf{R}_{\mathbf{n}_{2}}$ such that

$$
I\left(\mathbf{y}_{0} ; \mathbf{s}\right)=\frac{1}{2} \log _{2}|\mathbf{A}| \text { and } I\left(\mathbf{y}_{2} ; \mathbf{s}\right)=\frac{1}{2} \log _{2}|\mathbf{B}|,
$$

respectively. Furthermore, we can re-express (2) by using the matrix determinant formula in [11] as

$$
\begin{aligned}
I(\mathbf{y} ; \mathbf{s}) & =\frac{1}{2} \log _{2}|\mathbf{A}|+\frac{1}{2} \log _{2}\left|\mathbf{B}-\mathbf{C A}^{-1} \mathbf{D}\right| \\
& =\frac{1}{2} \log _{2}|\mathbf{B}|+\frac{1}{2} \log _{2}\left|\mathbf{A}-\mathbf{D B}^{-1} \mathbf{C}\right|
\end{aligned}
$$

The two previous equations can be expanded and further simplified by using the matrix determinant inverse lemma in [12] such that

$$
\begin{aligned}
I(\mathbf{y} ; \mathbf{s}) & =I\left(\mathbf{y}_{0} ; \mathbf{s}\right)+I\left(\widehat{\mathbf{y}}_{2} ; \mathbf{s}\right) \\
& =\frac{1}{2} \log _{2}\left|\mathbf{I}_{n}+\mathbf{R}^{\dagger} \mathbf{R}_{\mathbf{y}} \mathbf{R}\right|^{\prime}
\end{aligned}
$$

where

$$
\begin{array}{ll}
I\left(\widehat{\mathbf{y}}_{2} ; \mathbf{s}\right) & =\frac{1}{2} \log _{2}\left|\mathbf{I}_{r}+\mathbf{H}_{2} \mathbf{G}\left(\widehat{\mathbf{R}}_{\mathbf{y}_{1}}-\mathbf{R}_{\mathbf{n}_{1}}\right) \mathbf{G}^{\dagger} \mathbf{H}_{2}^{\dagger} \mathbf{R}_{\mathbf{n}_{2}}^{-1}\right| \\
\widehat{\mathbf{R}}_{\mathbf{y}_{1}} & =\mathbf{R}_{\mathbf{n}_{1}}+\mathbf{H}_{1} \mathbf{R} \widehat{\mathbf{A}}^{-1} \mathbf{R}^{\dagger} \mathbf{H}_{1}^{\dagger} \\
\widehat{\mathbf{A}} & =\mathbf{I}_{n}+\mathbf{R}^{\dagger} \mathbf{H}_{0}^{\dagger} \mathbf{R}_{\mathbf{n}_{0}}^{-1} \mathbf{H}_{0} \mathbf{R} \\
\mathbf{R}_{\mathbf{y}} & =\mathbf{H}_{0}^{\dagger} \mathbf{R}_{\mathbf{n}_{0}}^{-1} \mathbf{H}_{0}+\mathbf{H}_{1}^{\dagger} \mathbf{R}_{\mathbf{n}_{1}}^{-1}\left[\mathbf{I}_{q}-\mathbf{E}^{-1}\right] \mathbf{H}_{1} \\
\mathbf{E} & =\mathbf{I}_{q}+\left(\sigma_{1}^{2} / \sigma_{2}^{2}\right) \mathbf{G}^{\dagger} \mathbf{H}_{2}^{\dagger} \mathbf{H}_{2} \mathbf{G}
\end{array}
$$

Let us define the SNRs of the SN-DN, SN-RN and RNDN links as $\gamma_{0}=P_{1} / \sigma_{0}^{2}, \gamma_{1}=P_{1} / \sigma_{1}^{2}$ and $\gamma_{2}=P_{2} / \sigma_{2}^{2}$, respectively, where $P_{1}$ is the total transmit power of the SN, $P_{2}$ is the total transmit power of the RN and $P_{1}=P_{2}=1$ are normalized to unit power. According to the previous equations and $(6), I(\mathbf{y} ; \mathbf{s})$ can be approximated for three extreme SNR settings, as follows:

1) In the case that $\gamma_{0} \ll 1$, then $\mathbf{A} \simeq \mathbf{I}_{r}$, and hence, $I(\mathbf{y} ; \mathbf{s}) \simeq I\left(\mathbf{y}_{2} ; \mathbf{s}\right)$ is independent of $\mathbf{R}$.

2) In the case that $\gamma_{1} \ll 1$, then $\mathbf{R}_{\mathbf{y}} \simeq \mathbf{H}_{0}^{\dagger} \mathbf{R}_{\mathbf{n}_{0}}^{-1} \mathbf{H}_{0}^{\dagger}$, and hence, $I(\mathbf{y} ; \mathbf{s}) \simeq I\left(\mathbf{y}_{0} ; \mathbf{s}\right)$ is independent of $\mathbf{G}$.

3) In the case that $\gamma_{2} \gg \gamma_{1}$, then $\mathbf{E}^{-1} \simeq \mathbf{0}$, and hence, $I(\mathbf{y} ; \mathbf{s}) \simeq I\left(\widetilde{\mathbf{y}}_{0} ; \mathbf{s}\right)=\frac{1}{2} \log _{2} \mid \mathbf{I}_{n}+\mathbf{R}^{\dagger}\left(\mathbf{H}_{0}^{\dagger} \mathbf{R}_{\mathbf{n}_{0}}^{-1} \mathbf{H}_{0}^{\dagger}+\right.$ $\left.\mathbf{H}_{1}^{\dagger} \mathbf{R}_{\mathbf{n}_{1}}^{-1} \mathbf{H}_{1}\right) \mathbf{R} \mid$ is independent of $\mathbf{G}$.

4) Moreover, since $\mathbf{0} \preceq \widehat{\mathbf{A}}^{-1} \preceq \mathbf{I}_{n}$ and $\mathbf{0} \preceq \mathbf{E}^{-1} \preceq \mathbf{I}_{n}$, hence, $I\left(\mathbf{y}_{0} ; \mathbf{s}\right) \leq I(\mathbf{y} ; \mathbf{s}) \leq I\left(\mathbf{y}_{0} ; \mathbf{s}\right)+I\left(\mathbf{y}_{2} ; \mathbf{s}\right)$ and $I\left(\mathbf{y}_{0} ; \mathbf{s}\right) \leq I(\mathbf{y} ; \mathbf{s}) \leq I\left(\widetilde{\mathbf{y}}_{0} ; \mathbf{s}\right)$, respectively.

\section{RELATED WORKS}

\section{A. Maximization of the direct link MI via the precoder $\mathbf{R}$ at the $S N$}

In point-to-point (P2P) MIMO communication, i.e., if only the SN-DN link is active, the problem of finding the optimal precoding matrix $\mathbf{R}$ that maximizes $f(\mathbf{R})=2 I\left(\mathbf{y}_{0} ; \mathbf{s}\right)$ under a total power constraint, i.e.,

$$
\max _{\mathbf{R}} f(\mathbf{R}) \text { s.t. } \mathbf{R} \succeq \mathbf{0}, \operatorname{tr}\left(\mathbf{R} \mathbf{R}^{\dagger}\right) \leq P_{1},
$$

has been well investigated. On the one hand if the knowledge of $\mathbf{H}_{0}$ is not available at the SN, then $\mathbf{R}=\mathbf{R}_{\mathrm{EG}}=\sqrt{P_{1} / n} \mathbf{I}_{n}$ is the optimal solution [13]. On the other hand if $\mathbf{H}_{0}$ is known at the $\mathrm{SN}$, then the optimal solution is given by $\mathbf{R}=\mathbf{R}_{\mathrm{WF}_{0}}=\mathbf{V}_{0} \widetilde{\mathbf{R}}$ [14], where $\mathbf{V}_{0} \in \mathbb{C}^{n \times n}$ is an unitary matrix that contains the right singular vectors of $\mathbf{H}_{0}, \widetilde{\mathbf{R}}=$ $\operatorname{diag}\left(\sqrt{p_{1,1}}, \sqrt{p_{1,2}}, \ldots, \sqrt{p_{1, n}}\right)$ is a $n \times n$ diagonal matrix and the values of $p_{1, i}$ are obtained via a water-filling algorithm. Moreover, $\widetilde{\mathbf{R}} \simeq \mathbf{R}_{\mathrm{EG}}$ when $\gamma_{0} \gg 1$. 


\section{B. Maximization of the relay link MI via the precoder $\mathbf{G}$ at the $R N$}

In cooperative MIMO communication, it has recently been shown in [7] and [8] that the cooperative MI $I(\mathbf{y} ; \mathbf{x})$ can be increased by maximizing $f_{\mathbf{R}}(\mathbf{G})=I\left(\mathbf{y}_{2} ; \mathbf{x}\right)$, where the $\mathbf{R}$ matrix is a fixed parameter. The works in [7], [8] and [15] provide solutions for the following optimization problem

$$
\max _{\mathbf{G}} f_{\mathbf{R}}(\mathbf{G}) \text { s.t. } \mathbf{G} \succeq \mathbf{0}, \operatorname{tr}\left(\mathbf{G R}_{\mathbf{y}_{1}} \mathbf{G}^{\dagger}\right) \leq P_{2} .
$$

They solved this problem by considering that either the CSI of both the first and second hop channels, i.e., $\mathbf{H}_{1}$ and $\mathbf{H}_{2}$, [7], [8] or only the CSI of the first hop channel, i.e., $\mathbf{H}_{1}$, [7], [15] is available at the RN. In the former case, the optimal G matrix is given by $\mathbf{G}_{\text {opt }}=\mathbf{V}_{2} \widetilde{\mathbf{G}} \mathbf{U}^{\dagger}$ with $\mathbf{V}_{2}$ and $\mathbf{U} \in \mathbb{C}^{q \times q}$ are unitary matrices that contain the right singular vectors of $\mathbf{H}_{2}$ and the eigenvectors of $\mathbf{R}_{\mathbf{y}_{1}}$, respectively. In addition $\widetilde{\mathbf{G}}=\operatorname{diag}\left(\sqrt{p_{2,1}}, \sqrt{p_{2,2}}, \ldots, \sqrt{p_{2, q}}\right)$ is a $q \times q$ diagonal matrix, where the values of $p_{2, i}$ are obtained via Lagrangian optimization [16]. In the latter case, the optimal $\mathbf{G}$ matrix is yet to be found. In these works $\mathbf{R}=\mathbf{R}_{\mathrm{EG}}$.

\section{Maximization of the relay link MI via joint determination of the precoders $\mathbf{R}$ and $\mathbf{G}$}

Recently, we have investigated the following problem in [9]

$$
\begin{array}{rl}
\max _{\mathbf{R}, \mathbf{G}} & f(\mathbf{R}, \mathbf{G}) \\
\text { s.t. } & \mathbf{R} \succeq \mathbf{0}, \operatorname{tr}\left(\mathbf{R} \mathbf{R}^{\dagger}\right) \leq P_{1}, \\
& \mathbf{G} \succeq \mathbf{0}, \operatorname{tr}\left(\mathbf{G R}_{\mathbf{y}_{1}} \mathbf{G}^{\dagger}\right) \leq P_{2}
\end{array}
$$

where $f(\mathbf{R}, \mathbf{G})=I\left(\mathbf{y}_{2} ; \mathbf{s}\right)$, and design two algorithms that jointly optimize the two precoders $\mathbf{R}$ and $\mathbf{G}$ by assuming that either $\mathbf{H}_{1}$ and $\mathbf{H}_{2}$ are known at the $\mathrm{SN}$ and $\mathrm{RN}$ nodes or that $\mathbf{H}_{1}$ is known at the $\mathrm{SN}$ and $\mathbf{H}_{1}$ and $\mathbf{H}_{2}$ are known at the RN. Results have indicated that these two techniques perform similarly and outperform the techniques of Section III-B in terms of relay link MI. However, their cooperative MI performances are only better than those of Section III-B techniques when the direct link is weak, since their precoder $\mathbf{R}$ at the $\mathrm{SN}$ is designed regardless of the direct link quality.

\section{COOPERATIVE MUTUAL INFORMATION MAXIMIZATION}

In this section, we expand our work in [9] for designing the precoders $\mathbf{R}$ and $\mathbf{G}$ that maximize the cooperative MI, instead of the relay MI, when FCSI is available. In other words, we propose a novel power allocation method for finding a solution to the problem in (9) when $f(\mathbf{R}, \mathbf{G})=I(\mathbf{y} ; \mathbf{s})$.

This problem requires the optimization of two matrices at the same time and it cannot be solved by directly using classic convex optimization tools [16]. However, we can split the problem into two sub-problems by using the two distinct expressions of $I(\mathbf{y} ; \mathbf{s})$ in $(6)$, where $\mathbf{R}$ and $\mathbf{G}$ are assumed to be fixed in the first and second equations of (6), respectively. Then, we utilize a recursive approach to successively update $\mathbf{G}$ and $\mathbf{R}$ until the algorithm converges to a solution. Our algorithm is summarized in Algorithm 1 and 2.

\section{A. Initialization phase}

In the initialization phase of our algorithm, we aim at finding the $\mathbf{G}$ matrix that maximizes $I\left(\widehat{\mathbf{y}}_{2} ; \mathbf{s}\right)$ in (6) when $\mathbf{R}$ is fixed and such that $\mathbf{R}=\mathbf{R}_{\mathrm{EG}}$. Notice that $\mathbf{G}=\mathbf{G}_{\mathrm{opt}}$ when the direct link is weak in comparison with the relay link, since $I\left(\mathbf{y}_{2} ; \mathbf{s}\right)$ and $I\left(\widehat{\mathbf{y}}_{2} ; \mathbf{s}\right)$ are equivalent in this case.

The $\mathbf{G}$ matrix that maximizes $I(\mathbf{y} ; \mathbf{s})$ for a fixed $\mathbf{R}$ can be obtained by using the first equation of (6) and solving the same problem as in (8) but for $f_{\mathbf{R}}(\mathbf{G})=I\left(\widehat{\mathbf{y}}_{2} ; \mathbf{s}\right)$. The term $I\left(\widehat{\mathbf{y}}_{2} ; \mathbf{s}\right)$ in (6) can be re-expressed as

$$
I\left(\widehat{\mathbf{y}}_{2} ; \mathbf{s}\right)=\frac{1}{2} \log _{2}\left|\frac{\sigma_{2}^{2} \mathbf{I}_{r}+\mathbf{H}_{2} \mathbf{G} \widehat{\mathbf{R}}_{\mathbf{y}_{1}} \mathbf{G}^{\dagger} \mathbf{H}_{2}^{\dagger}}{\sigma_{2}^{2} \mathbf{I}_{r}+\mathbf{H}_{2} \mathbf{G} \mathbf{R}_{\mathbf{n}_{1}} \mathbf{G}^{\dagger} \mathbf{H}_{2}^{\dagger}}\right| .
$$

The matrix $\mathbf{H}_{2}$ can be decomposed via singular value decomposition (SVD) as $\mathbf{H}_{2}=\mathbf{U}_{2} \widehat{\Omega}^{\frac{1}{2}} \mathbf{V}_{2}^{\dagger}$, where $\mathbf{U}_{2} \in \mathbb{C}^{r \times r}$ and $\mathbf{V}_{2} \in \mathbb{C}^{q \times q}$ are unitary matrices, $\widehat{\Omega} \in \mathbb{C}^{r \times q}$ is a rectangular diagonal matrix, and $\boldsymbol{\Omega}=\widehat{\boldsymbol{\Omega}}^{\frac{1}{2}} \widehat{\boldsymbol{\Omega}}^{\frac{1}{2} \dagger}$ is a $r \times r$ diagonal matrix with diagonal elements $\omega_{i} \in \mathbb{R}_{+}$, i.e., $\mathbb{R}_{+}=\{x \in \mathbb{R} \mid x \geq 0\}$, which are sorted in descending order as in [8]. Notice that $\omega_{i} \neq 0$ for $i \in\left[1, N_{\omega}\right]$ and that $\omega_{i}=0$ for $i \in\left[N_{\omega}+1, r\right]$, with $N_{\omega}=\min \{r, q\}$. Similarly, the matrices $\widehat{\mathbf{R}}_{\mathbf{y}_{1}}$ and $\mathbf{R}_{\mathbf{y}_{1}}$ can be decomposed via eigenvalue decomposition (EVD) as $\widehat{\mathbf{R}}_{\mathbf{y}_{1}}=\widehat{\mathbf{U}} \boldsymbol{\Delta} \widehat{\mathbf{U}}^{\dagger}$ and $\mathbf{R}_{\mathbf{y}_{1}}=\mathbf{U} \boldsymbol{\Lambda} \mathbf{U}^{\dagger}$, respectively, where $\widehat{\mathbf{U}} \in \mathbb{C}^{q \times q}$ is a unitary matrix and $\boldsymbol{\Delta}$ and $\boldsymbol{\Lambda}$ are $q \times q$ diagonal matrices with diagonal elements $\delta_{i} \in \mathbb{R}_{+}$and $\lambda_{i} \in \mathbb{R}_{+}$, respectively, which are sorted in descending order [7]. In the case that $\mathbf{U}=\widehat{\mathbf{U}}$, we can set $\mathbf{G}=\mathbf{G}_{\text {opt }}$ and simplify (10) as

$I\left(\widetilde{\mathbf{y}}_{2} ; \mathbf{s}\right)=\frac{1}{2} \sum_{i=1}^{N_{\omega}} \log _{2}\left(\sigma_{2}^{2}+p_{2, i} \omega_{i} \delta_{i}\right)-\log _{2}\left(\sigma_{2}^{2}+p_{2, i} \omega_{i} \sigma_{1}^{2}\right)$.

Then, the values of $p_{2, i}$ that maximize $I\left(\widetilde{\mathbf{y}}_{2} ; \mathbf{s}\right)$ are obtained by solving the following simplified problem

$$
\max _{\mathbf{p}_{2}} I\left(\widetilde{\mathbf{y}}_{2} ; \mathbf{s}\right) \text { s.t. } p_{2, i} \geq 0 ; \mathrm{P}_{\mathrm{c}}: \sum_{i=1}^{N_{\omega}} p_{2, i} \lambda_{i} \leq P_{2},
$$

where $\mathbf{p}_{2}=\left\{p_{2,1}, p_{2,2}, \ldots, p_{2, q}\right\}$. The optimum solution for this modified problem is obtained by Lagrangian optimization, such that $p_{2, i}(\mu)=$

$$
\left[\frac{-\sigma_{2}^{2}\left(\delta_{i}+\sigma_{1}^{2}\right)+\sqrt{\sigma_{2}^{2}\left(\delta_{i}-\sigma_{1}^{2}\right)\left[\sigma_{2}^{2}\left(\delta_{i}-\sigma_{1}^{2}\right)+4 \mu \omega_{i}\left(\delta_{i} / \lambda_{i}\right)\right]}}{2 \sigma_{1}^{2} \omega_{i} \delta_{i}}\right],
$$

where $[x]_{+}=\max \{0, x\}$ and $\mu \geq 0$ is the Lagrange multiplier that needs to be tuned for satisfying the power constraint $\mathrm{P}_{\mathrm{c}}$ in (12). The starting value for $\mu$ is $\mu_{\min }=$ $\max _{i \in\left[1, N_{\omega}\right]}\left\{\sigma_{1}^{2} \sigma_{2}^{2} \lambda_{i} /\left[\left(\delta_{i}-\sigma_{1}^{2}\right) \omega_{i}\right]\right\}$; then $\mu$ is be updated by using the Newton-Raphson method [17] until $\mu^{\star}$ is obtained. The value of $\mu^{\star}$ must fulfill the following inequality $g\left(\mu^{\star}\right)<$ $\epsilon$, with $g(\mu)=\sum_{i=1}^{N_{\omega}} p_{2, i}(\mu) \lambda_{i}-P_{2}$ and $\epsilon \ll 1$.

The previous algorithm can only be implemented if $\mathbf{U}=$ $\widehat{\mathbf{U}}$, which will not be the case for any random $\mathbf{R}$ matrix. Let $\mathbf{V}_{1} \in \mathbb{C}^{n \times n}$ be an unitary matrix that contains the right singular vectors of $\mathbf{H}_{1}$, we can re-expressed $\mathbf{R}_{\mathbf{y}_{1}}=\mathbf{R}_{\mathbf{n}_{1}}+\mathbf{H}_{1} \mathbf{V}_{1}\left(\mathbf{V}_{1}^{\dagger} \mathbf{R} \mathbf{R}^{\dagger} \mathbf{V}_{1}\right) \mathbf{V}_{1}^{\dagger} \mathbf{H}_{1}^{\dagger}$ and $\widehat{\mathbf{R}}_{\mathbf{y}_{1}}=\mathbf{R}_{\mathbf{n}_{1}}+$ 
$\mathbf{H}_{1} \mathbf{V}_{1}\left(\mathbf{V}_{1}^{\dagger} \mathbf{R} \widehat{\mathbf{A}}^{-1} \mathbf{R}^{\dagger} \mathbf{V}_{1}\right) \mathbf{V}_{1}^{\dagger} \mathbf{H}_{1}^{\dagger}$. Clearly, if both $\left(\mathbf{V}_{1}^{\dagger} \mathbf{R} \mathbf{R}^{\dagger} \mathbf{V}_{1}\right)$ and $\left(\mathbf{V}_{1}^{\dagger} \mathbf{R} \widehat{\mathbf{A}}^{-1} \mathbf{R}^{\dagger} \mathbf{V}_{1}\right)$ are diagonal matrices then $\mathbf{U}=\widehat{\mathbf{U}}$. This condition can simply be fulfilled by setting $\mathbf{R}=$ $\mathbf{V}_{1} \widetilde{\mathbf{R}} \mathbf{U}_{a}^{\dagger}$, where $\mathbf{U}_{a} \in \mathbb{C}^{n \times n}$ is an unitary matrix that contains the left singular vectors of $\widehat{\mathbf{A}}^{-1}$. However, $\widehat{\mathbf{A}}^{-1}$ is also dependent of $\mathbf{R}$ and $\mathbf{R}$ must be set in $\widehat{\mathbf{A}}^{-1}$ prior to the computation of $\mathbf{U}_{a}$. Therefore, this problem is equivalent to find a matrix $\mathbf{U}_{a}$ such that $\left[\mathbf{U}_{a}, \mathbf{X}, \mathbf{Y}\right]=\operatorname{svd}\left\{\left(\mathbf{I}_{n}+\right.\right.$ $\left.\left.\left(\mathbf{V}_{1} \widetilde{\mathbf{R}} \mathbf{U}_{a}^{\dagger}\right)^{\dagger} \mathbf{H}_{0}^{\dagger} \mathbf{R}_{\mathbf{n}_{0}}^{-1} \mathbf{H}_{0}\left(\mathbf{V}_{1} \widetilde{\mathbf{R}} \mathbf{U}_{a}^{\dagger}\right)\right)^{-1}\right\}$, where $\operatorname{svd}\{\mathbf{Z}\}$ is a function that returns the matrices $\mathbf{U}_{a}, \mathbf{X}$ and $\mathbf{Y}$ that contain the left singular vectors, eigenvalues and right singular vectors of $\mathbf{Z}$, respectively. Finding a solution to this problem is outside the scope of this paper and instead we set $\mathbf{R}=\overline{\mathbf{R}}=\mathbf{V}_{1} \widetilde{\mathbf{R}}$ in $\widehat{\mathbf{A}}^{-1}$. By approximating $\widehat{\mathbf{R}}_{\mathbf{y}_{1}}=$ $\mathbf{R}_{\mathbf{n}_{1}}+\mathbf{H}_{1} \mathbf{R}\left(\mathbf{I}_{n}+\mathbf{R}^{\dagger} \mathbf{H}_{0}^{\dagger} \mathbf{R}_{\mathbf{n}_{0}}^{-1} \mathbf{H}_{0} \mathbf{R}\right)^{-1} \mathbf{R}^{\dagger} \mathbf{H}_{1}^{\dagger}$ with $\widehat{\mathbf{R}}_{\mathbf{y}_{1}}=$ $\mathbf{R}_{\mathbf{n}_{1}}+\mathbf{H}_{1} \mathbf{R}\left(\mathbf{I}_{n}+\overline{\mathbf{R}}^{\dagger} \mathbf{H}_{0}^{\dagger} \mathbf{R}_{\mathbf{n}_{0}}^{-1} \mathbf{H}_{0} \overline{\mathbf{R}}\right)^{-1} \mathbf{R}^{\dagger} \mathbf{H}_{1}^{\dagger}$, we simplify the optimization problem, which becomes equivalent to (12). In addition, we narrow the search down to the $\mathbf{G}$ matrices of the $\mathbf{G}_{\text {opt }}$ type, which are optimal if the direct link is weaker than the relay link. At the end of the initialization phase, we set $\mathbf{G}$ in $\mathbf{R}_{\mathbf{y}}$ and evaluate $y^{(0)}=I(\mathbf{y} ; \mathbf{s})$ by using the second equation of (6) with either $\mathbf{R}=\mathbf{V}_{1} \widetilde{\mathbf{R}} \mathbf{U}_{a}^{\dagger}$ or $\mathbf{R}=\mathbf{V}_{1} \widetilde{\mathbf{R}}$, since both these $\mathbf{R}$ matrices provide the same result for $I(\mathbf{y} ; \mathbf{s})$.

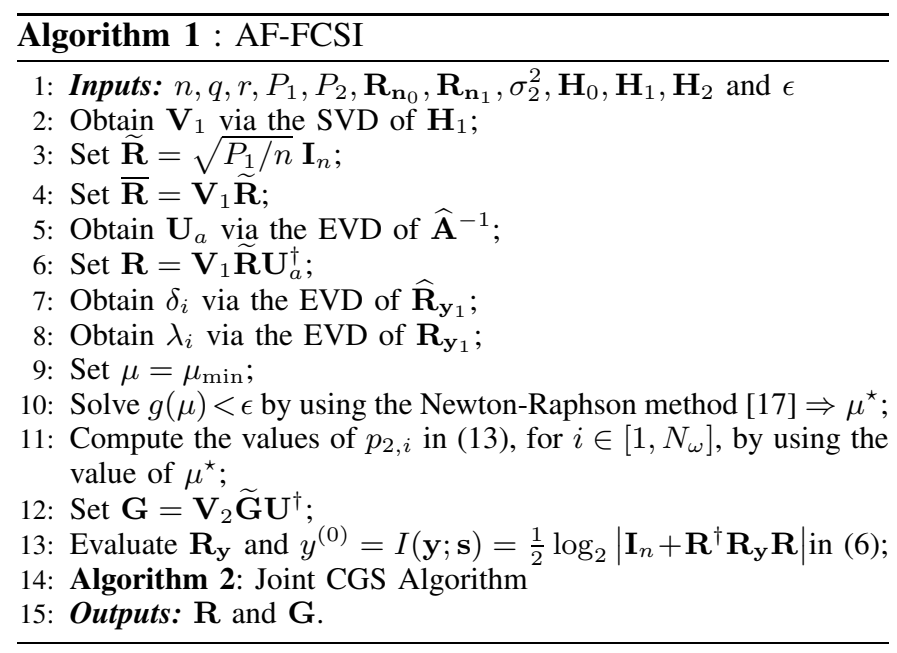

\section{B. Recursive phase}

In the recursive phase of our algorithm, we utilize a joint constrained gradient search (CGS) algorithm for first finding the matrix $\mathbf{R}$ that maximizes $I(\mathbf{y} ; \mathbf{s})$ when $\mathbf{G}$ is fixed; see steps 4-9 of Algorithm 2. Then, we compute $\mathbf{R}_{\mathbf{y}_{1}}$ and $\widehat{\mathbf{R}}_{\mathbf{y}_{1}}$ according to the new version of $\mathbf{R}$, i.e., $\widehat{\mathbf{R}}$, and modify $\mathbf{G}$ to ensure that $\operatorname{tr}\left(\mathbf{G R}_{\mathbf{y}_{1}} \mathbf{G}^{\dagger}\right) \leq P_{2}$; see steps 10-13 of Algorithm 2. Next, we aim at finding the matrix $\mathbf{G}$ that maximizes the first equation of (6) when $\mathbf{R}$ is fixed; see steps 14-19 of Algorithm 2. We compute $\widehat{\mathbf{R}}_{\mathbf{y}}$ according to the new version of $\mathbf{G}$, i.e., $\widehat{\mathbf{G}}$ and evaluate $y^{(m)}=\frac{1}{2} \log _{2}\left|\mathbf{I}_{n}+\widehat{\mathbf{R}}^{\dagger} \widehat{\mathbf{R}}_{\mathbf{y}} \widehat{\mathbf{R}}\right|$ at the $m$-th iteration. Finally, $y^{(m)}$ is compared against $y^{(m-1)}$ and the algorithm proceeds until $\left|y^{(m)}-y^{(m-1)}\right|<\varepsilon$ or the number of iteration $m$ is above $1 / \varepsilon$.

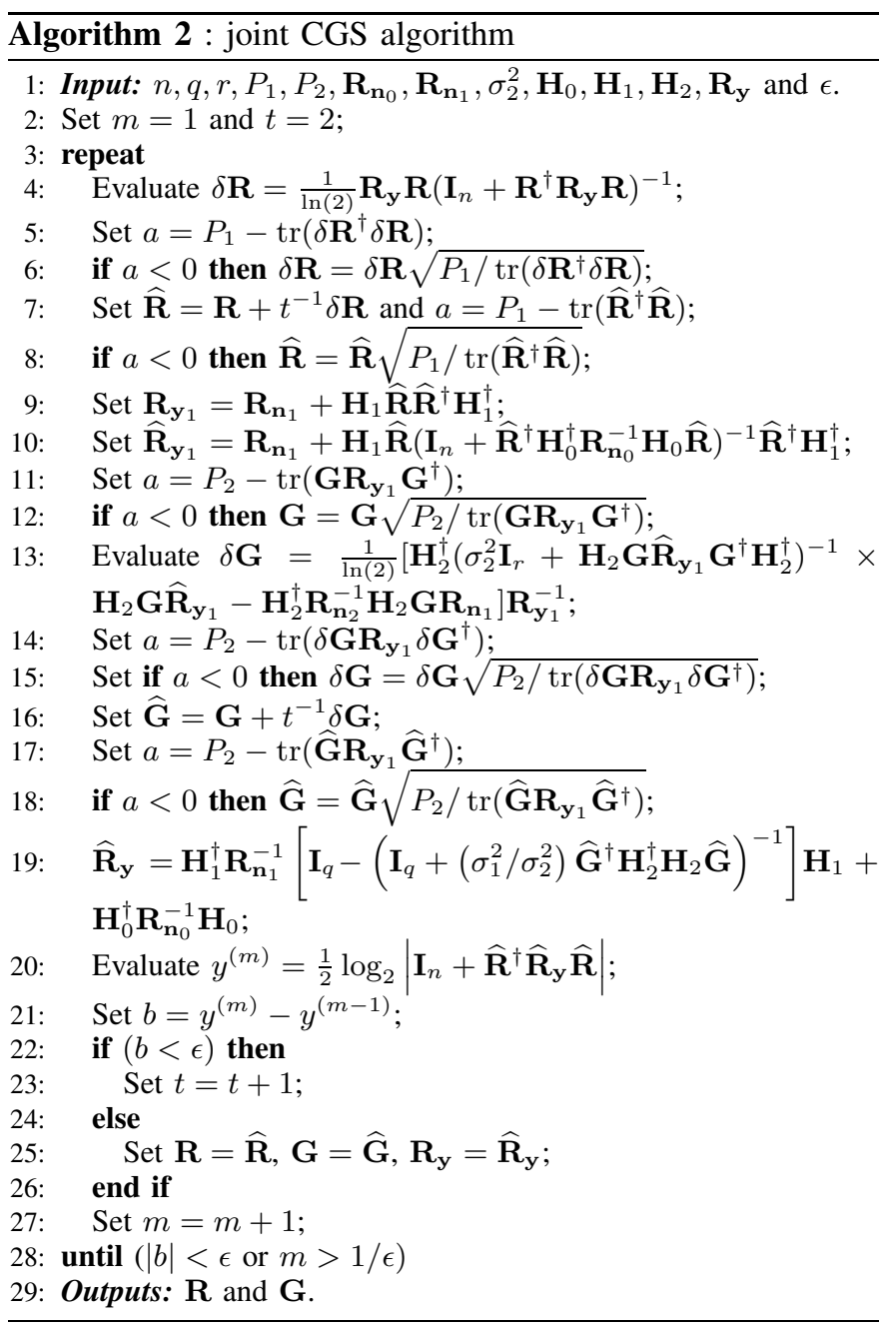

\section{NUMERICAL RESUlts AND Discussion}

In this section, our novel AF-FCSI power allocation method is compared in terms of cooperative MI against the methods in [8] and [9], which are here refereed as AF- [8] and AF[9], respectively, for various link SNR conditions. We also plot $I(\mathbf{y} ; \mathbf{s})=2 I\left(\mathbf{y}_{0} ; \mathbf{s}\right)$ for $\mathbf{R}=\mathbf{R}_{\mathrm{WF}_{0}}$, which is the MI of a P2P MIMO system when the CSI is available at the $\mathrm{SN}$. As we already stated, acquiring FCSI at the SN and RN nodes is impractical. A practical solution can be to acquire the SN-RN and SN-DN link CSI at the SN and the SNRN and RN-DN link CSI at the RN. In this case, power allocation can independently be made at the $\mathrm{SN}$ and $\mathrm{RN}$ by solving the problem in (7) but for $f(\mathbf{R})=I\left(\widetilde{\mathbf{y}}_{0} ; \mathbf{s}\right)$, where the optimal solution is $\mathbf{R}_{\mathrm{WF}_{1}}=\widehat{\mathbf{W}} \widetilde{\mathbf{R}}$ with $\widehat{\mathbf{W}} \in \mathbb{C}^{n \times n}$ being an unitary matrix that contains the eigenvectors of $\left(\mathbf{H}_{0}^{\dagger} \mathbf{R}_{\mathbf{n}_{0}}^{-1} \mathbf{H}_{0}^{\dagger}+\mathbf{H}_{1}^{\dagger} \mathbf{R}_{\mathbf{n}_{1}}^{-1} \mathbf{H}_{1}\right)$, and by using the AF- [8] method, respectively. We denote this method as AF-Total CSI (TCSI) and include it in the following performance comparison.

In our simulations, a single-tap i.i.d. Rayleigh fading channel is assumed for each of the SN-DN, SN-RN and RN-DN links. Moreover, the parameter $\epsilon$, which is used for fine-tuning the accuracy of our AF-FCSI algorithm, is set to $\epsilon=10^{-4}$. 


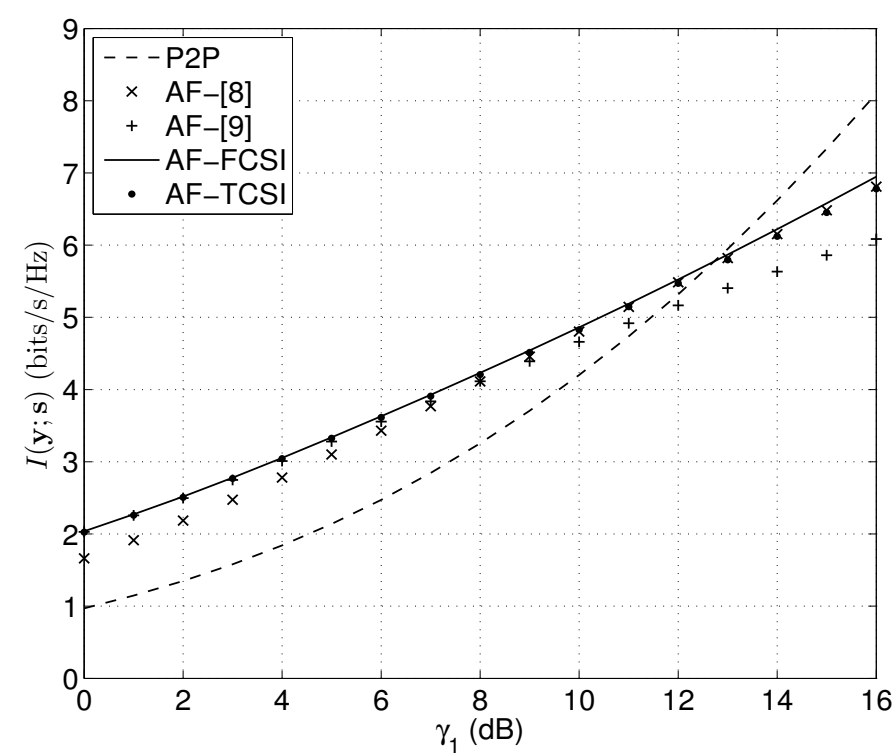

Fig. 2. Cooperative MI performance of various power allocation methods against $\gamma_{1} \mathrm{~dB}$ for $\gamma_{0}=\left(\gamma_{1}-10\right) \mathrm{dB}, \gamma_{2}=10 \mathrm{~dB}$ and $n=q=r=4$.

In Fig. 2, we compare the cooperative MI performance of various power allocation algorithms for $n=q=r=4$, $\gamma_{0}=\left(\gamma_{1}-10\right) \mathrm{dB}$ and $\gamma_{2}=10 \mathrm{~dB}$. The results first show that the range of $\gamma_{1} \mathrm{~dB}$ for which cooperative communication outperforms P2P communication is limited to $13 \mathrm{~dB}$. Since $\gamma_{2}$ is fixed, the RN-DN acts as a bottleneck and the relay link MI does not increase as fast as the direct link MI for $\gamma_{1}>13 \mathrm{~dB}$. The results also show the advantage of source power allocation when $\gamma_{1}$ is low, since our AF-FCSI, AF-TCSI and AF- [9] methods outperform the AF- [8] technique in this case. The performance gain decreases as $\gamma_{0}$ and $\gamma_{1}$ grows, since EG power allocation is the best policy at the SN when both $\gamma_{0}$ and $\gamma_{1} \gg 1$. Moreover, the comparison of the AF- [8] with the AF- [9] scheme emphasizes that $\gamma_{0}$ has to be fairly low for the AF- [9] method to outperform the AF- [8] scheme.

In Fig. 3, we compare the same algorithms but for $\gamma_{0}=0$ $\mathrm{dB}$ instead of $\gamma_{0}=\gamma_{1}-10 \mathrm{~dB}$. The results show again the advantage of our AF-FCSI and AF-TCSI methods against the AF- [8] scheme at low $\gamma_{1} \mathrm{~dB}$, but actually in the range of $\gamma_{1} \mathrm{~dB}$ where cooperative communication is not efficient. The three previously mentioned methods perform similarly for $\gamma_{1} \in[7,20] \mathrm{dB}$ and then the performance gap between our AF-FCSI algorithm and the other methods increases as $\gamma_{1} \mathrm{~dB}$ increases. In the latter case, the RN-DN link acts as a bottleneck and extra cooperative MI performance can be achieved by properly balancing the power allocated between the relay and direct links, i.e., reducing the allocated power of the relay link and increasing the allocated power of the direct link. The results indicate that only our AF-FCSI can properly balance the power distribution by taking advantage of the joint power allocation. The result of the AF- [9] technique indicates that it is preferable to use an EG power allocation than a power allocation that solely takes into account the SNRN link CSI when $\gamma_{0}$ and $\gamma_{1}$ are similar. Moreover, as $\gamma_{1}$

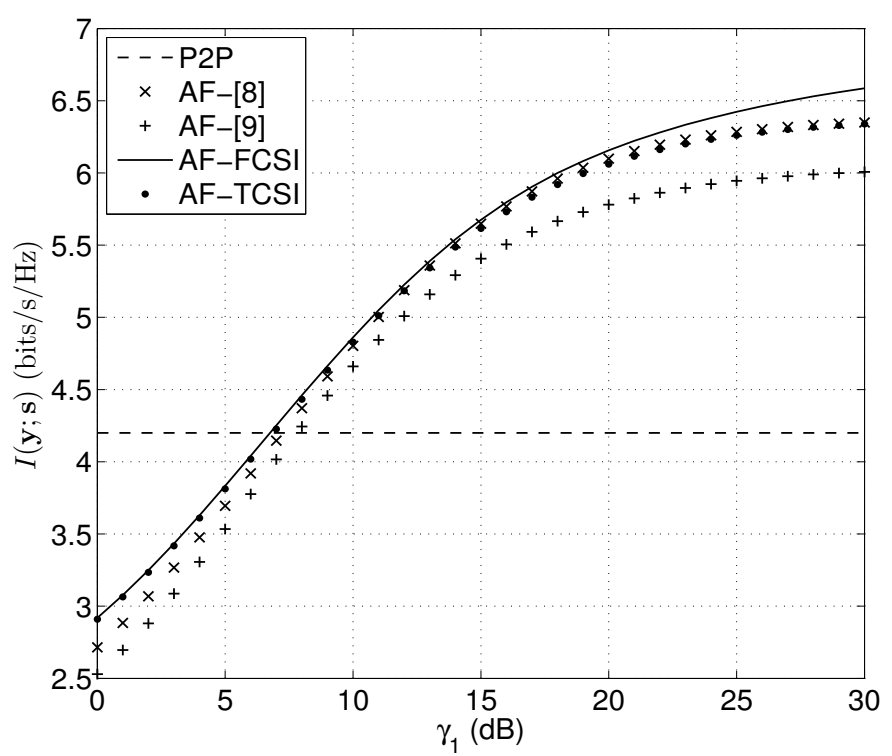

Fig. 3. Cooperative MI performance of various power allocation methods against $\gamma_{1} \mathrm{~dB}$ for $\gamma_{0}=0 \mathrm{~dB}, \gamma_{2}=10 \mathrm{~dB}$ and $n=q=r=4$.

grows, this technique gives more weight to the relay link and if the RN-DN link is weaker than the SN-RN link then more performance degradation will occur in comparison with the AF- [8] technique.

In Fig. 4, we compare the same algorithms for $n=q=$ $r=4, \gamma_{0}=0 \mathrm{~dB}$ and $\gamma_{1}=10 \mathrm{~dB}$. We also depict the performance bounds $\mathrm{B} 1$ and $\mathrm{B} 2$, where $I(\mathbf{y} ; \mathbf{s})=I\left(\widetilde{\mathbf{y}}_{0} ; \mathbf{s}\right)$ for $\mathbf{R}_{\mathrm{WF}_{1}}$ and $\mathbf{R}_{\mathrm{EG}}$, respectively. The results show that the AFFCSI, AF-TCSI and AF- [8] methods perform similarly at low SNRs. As $\gamma_{2}$ increases, the impact of power allocation at the $\mathrm{RN}$ on $I(\mathbf{y} ; \mathbf{s})$ diminishes and the performance of the various methods get closer to the bound B1 and B2. This result is consistent with the approximation 3) at the end of Section II. The difference between B1 and B2, i.e., acquiring or not the CSI at the source, is only of $0.1 \mathrm{bits} / \mathrm{sHz}$, which is very small in comparison with the extra complexity that is involved for acquiring the SN-RN and SN-DN link CSI at the SN.

In Fig. 5, we plot $G_{I}=I(\mathbf{y} ; \mathbf{s})_{\mathrm{AF}-\mathrm{FCSI}}-I(\mathbf{y} ; \mathbf{s})_{\mathrm{AF}-[8]}$ against $\gamma_{1} \mathrm{~dB}$ and $\gamma_{2} \mathrm{~dB}$ for $n=q=r=4$ and $\gamma_{0}=\left(\gamma_{1}-\right.$ 10) dB. $G_{I}$ is the performance gain in terms of cooperative MI that our AF-FCSI method achieves when compared with the AF- [8] technique. On the graph, the light grey shaded area on the left represents the range of $\gamma_{1}$ and $\gamma_{2}$ SNRs for which P2P outperforms cooperative communication. Outside this SNR area, $G_{I}$ increases as $\gamma_{1}$ increases from -10 to -2 $\mathrm{dB}$ and then decreases until $\gamma_{1}=10 \mathrm{~dB}$ regardless of $\gamma_{2}$. The gain $G_{I}$ starts to vary in function of $\gamma_{2}$ only when $\gamma_{1}>10$ $\mathrm{dB}$. This graph mainly points out that $G_{I}>0$ and, hence, our AF-FCSI method always outperforms the AF- [8] scheme.

Overall, the performance analysis demonstrates that our AF-FCSI method clearly outperforms the other presented techniques and, thus, its performances act as upper bounds for these techniques. However, the performance gain of our joint power allocation technique against the other techniques 


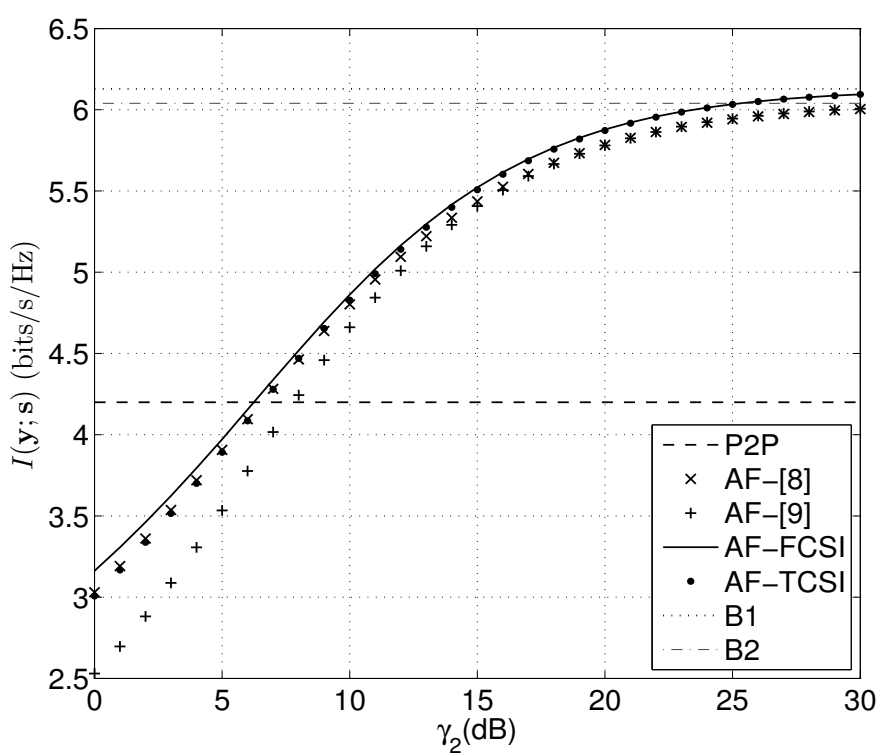

Fig. 4. Cooperative MI performance of various power allocation methods against $\gamma_{2} \mathrm{~dB}$ for $\gamma_{0}=0 \mathrm{~dB}, \gamma_{1}=10 \mathrm{~dB}$ and $n=q=r=4$.

remains relatively small, except when $\gamma_{1} \gg \gamma_{2}$, in comparison with the complexity that is required for acquiring FCSI at the RN and SN nodes. To this end, the AF-TCSI method can be a practical alternative since it can provide similar performance as the AF-FCSI scheme, especially when $\gamma_{0}$ is low. Finally, the results also indicate that the impact of power allocation at the $\mathrm{RN}$ diminishes as the $\mathrm{RN}$ gets closer to the $\mathrm{DN}$.

\section{CONCLUSION}

in this paper, we have proposed a joint power allocation algorithm for maximizing the cooperative $\mathrm{MI}$ of $\mathrm{AF}$ system by considering that FCSI is available at the $\mathrm{SN}$ and $\mathrm{RN}$ nodes. The joint power allocation problem requires the optimization of two matrices at the same time and it cannot be solved by directly using classic convex optimization tools. However, we have shown how to split the main problem into two sub-problems by expressing the cooperative MI in two distinct ways. We also proposed a simplified version of our scheme. Then, we have compared our novel schemes with other existing power allocation methods for $\mathrm{AF}$ in various link SNR configurations. The results show that our AFFCSI clearly outperforms the other techniques. However, its performance gain remains relatively small in comparison with the complexity it incurs. It turns out that practical scheme such as the one in [8] or our AF-TCSI method can be used to obtain close to AF-FCSI performance for most of the link SNR configurations where cooperative communication is more efficient than P2P communication.

\section{REFERENCES}

[1] J. N. Laneman and G. W. Wornell, "Distributed Space-Time-Coded Protocols for Exploiting Cooperative Diversity in Wireless Networks," IEEE Trans. Inform. Theory, vol. 49, no. 10, pp. 2415-2425, Oct. 2003.

[2] A. Sendonaris, E. Erkip, and B. Aazhang, "User Cooperation Diversity Part I - System Description,” IEEE Trans. Commun., vol. 51, no. 11, pp. 1927-1938, Nov. 2003.

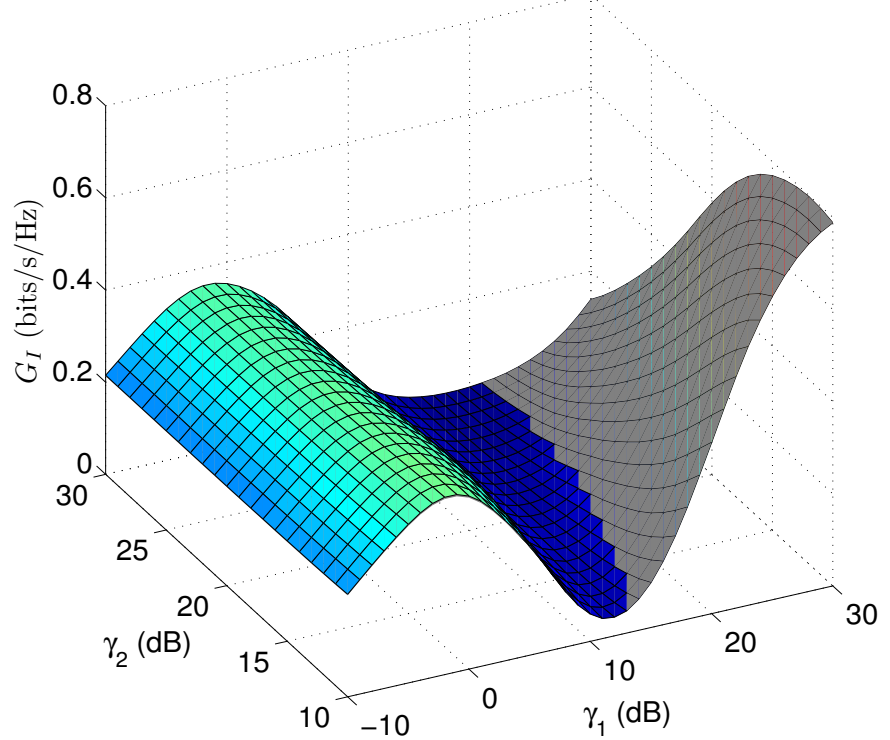

Fig. 5. Cooperative MI gain of our AF-FCSI method vs.the AF- [8] scheme for $\gamma_{0}=\left(\gamma_{1}-10\right) \mathrm{dB}$ and $n=q=r=4$.

[3] — , "User Cooperation Diversity Part II - Implementation Aspects and Performance Analysis," IEEE Trans. Commun., vol. 51, no. 11, pp. 1939-1948, Nov. 2003.

[4] M. Janani, A. Heday, T. Hunter, and A. Nosratinia, "Coded Cooperation in Wireless Communications: Space-Time Transmission and Iterative Decoding," IEEE Trans. Signal Processing, vol. 52, no. 2, pp. 362-371, Feb. 2004

[5] A. Nosratinia, T. E. Hunter, and A. Hedayat, "Cooperative Communication in Wireless Networks," IEEE Commun. Mag., vol. 42, no. 10, pp. 74-80, Oct. 2004.

[6] J. N. Laneman, D. N. C. Tse, and G. W. Wornell, "Cooperative Diversity in Wireless Networks: Efficient Protocols and Outage Behavior," IEEE Trans. Inform. Theory, vol. 50, no. 12, pp. 3062-3080, Dec. 2004.

[7] Y. Fan and J. Thompson, "MIMO Configurations for Relay Channels: Theory and Practice," IEEE Trans. Wireless Commun., vol. 6, no. 5, pp. 1774-1786, May 2007.

[8] O. Muñoz-Medina, J. Vidal, and A. Agustn, "Linear Transceiver Design in Nonregenerative Relays with Channel State Information," IEEE Trans. Signal Processing, vol. 55, no. 6, pp. 2593-2604, June 2007.

[9] F. Héliot, R. Hoshyar, and R. Tafazolli, "Power Allocation for Nonregenerative Cooperative MIMO Communication under Various Levels of Channel State Information," in Proc. ICT Mobile Summit, Santander, Spain, June 2009.

[10] T. M. Cover and J. A. Thomas, Elements of Information Theory. NewYork, USA: ed. Wiley, 1991.

[11] "Imperial College Website, Block Matrix Determinant," [Online]. Available: http://www.ee.ic.ac.uk/hp/staff/dmb/matrix/ proof003.html.

[12] "Wikipedia Website," [Online]. Available: http://en.wikipedia.org/wiki/Matrix_determinant_lemma.

[13] G. J. Foschini, "On Limits of Wireless Personal Communications in a Fading Environment when using Multiple Antennas," Wireless Pers. Commun., vol. 6, pp. 311-315, Mar. 1998.

[14] G. G. Raleigh and J. M. Cioffi, "Spatio-Temporal Coding for Wireless Communication," IEEE Trans. Commun., vol. 46, pp. 357-366, Mar. 1998.

[15] F. Héliot, S. Fazel, R. Hoshyar, and R. Tafazolli, "Receive Knowledge Only Power Allocation for Nonregenerative Cooperative MIMO Communication," in Proc. IEEE SPAWC, Perugia, Italy, June 2009.

[16] S. Boyd and L. Vandenberghe, Convex Optimization. Cambridge, UK: Cambridge Univ. Press, 2004.

[17] "Wolfram Mathworld Website," [Online]. Available: http://mathworld.wolfram.com/NewtonsMethod.html. 